\title{
Algorithm for received signal in multipath propagation conditions
}

\author{
Dmitry Chernyshov ${ }^{1, *}$ \\ ${ }^{1}$ Don State Technical University, 344000 Rostov-on-Don, Russia
}

\begin{abstract}
This article discusses the received signal reception algorithm under conditions of multipath propagation of radio waves. As well as an analytical expression of the effectiveness of this algorithm is derived from the point of view of SNR.
\end{abstract}

\section{Introduction}

In recent years, radio systems are used widely in various fields of science and technology. This leads to their development and progress. However, the prevalence and widespread use of radio systems leads to a lack of frequency resource. Many systems and protocols operate on the same frequency, which leads to the occurrence of active interference and difficulty in receiving a signal. Increase in operating frequencies, aimed at increasing the data rate leads to a decrease in the service area and a greater influence of multiple reflections, which is most characteristic for the urban environment. This phenomenon is on the one hand, facilitates the transfer signal in shading, on the other, leads to intersymbol interference and as a result the occurrence of bit errors.

Since the main task of radio engineering is reliable transmission of messages at a distance from the transmitter to the receiver, it is necessary to develop methods that increase the efficiency of the wireless data transmission system, in a medium with reflections, in conditions of active interference. This problem is acute in mind the increasing prevalence of radio systems, data growth, and as a consequence of the need to increase the data rate.

\section{Algorithm}

As was shown in $[1,2]$, the signal in urban conditions spreads in many ways, which leads to the formation of copies of the same signal, but coming from different directions, delayed for some time and weakened by a certain amount. This in turn leads to bit errors [3].

To improve the efficiency of the receiver and eliminate the problems described above, an algorithm has been developed, shown in Figure 1

\footnotetext{
* Corresponding author: dimcher@inbox.ru
} 


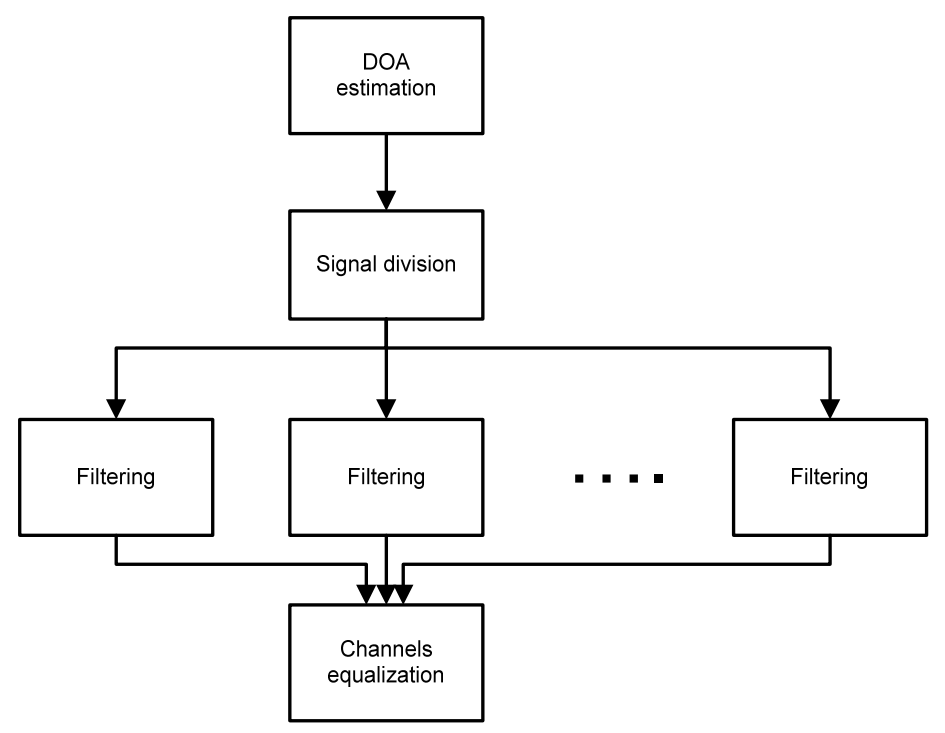

Fig. 1. Algorithm flowchart.

The algorithm is implemented as follows:

1. At the first stage of the algorithm, the direction of the signal sources is determined. Super-resolution methods [4-7] are best suited for this.

2.In accordance with certain directions of the signal, the weights of the antenna array (AA) are formed to direct the directional characteristic of the AA to the path corresponding to the signal. Thus, virtual AAs are formed and the signals are divided into different channels.

3. The signal in each channel is filtered.

4. Channels are aligned with each other to compensate for phase delays between signals. Thereafter, the signals are summed.

\section{Algorithm efficiency}

Suppose that $n$ signals from different directions are input to the AA. For each of the signals formed its own channel. On each of the receive channels of additive Gaussian noise, because of the differences and independence of random processes acting on the receive channels, we can talk about the noise uncorrelated $[8,9]$. Signal band is the same and is limited to the bandwidth of the receiver.

In general, without taking into account the filtering of each channel, it can be written that the total signal-to-noise ratio can be written by the following formula:

$$
S N R=\frac{\sum_{i=1}^{n} S_{i}}{\sum_{i=1}^{n} N_{i}},
$$

where $S_{i}$ - power of the $i$-th signal in the $i$-th channel; $N_{i}$ - noise power affecting the $i$ th channel.

Assume that the noise power in each channel is the same, and each term $\frac{S}{N}>0$. 
Then equation (1) can be simplified as:

$$
S N R=\frac{\sum_{i=1}^{n} S_{i}}{n N} .
$$

Considering that when adding signals from different sources, in the case of coincidence of the amplitude and phase components of the signal spectra, due to the equalization of the channels, it is necessary to add all the spectral components of the signals, then:

$$
S N R=\frac{\sum_{i=1}^{n} S_{0}}{n N}=\frac{\left(\sum_{i=1}^{n} A_{i}\right)^{2}}{n N} .
$$

We take for the minimum signal level in the channels - $A_{0}$, then the level of the $i$-th signal will be $A_{i}=k_{i} A_{0}$, where $k_{i}$ is the weight coefficient of the $i$-th signal. Given this, the expression (3) can be rewritten in the form:

$$
S N R=\frac{\left(\sum_{i=1}^{n} k_{i} A_{0}\right)^{2}}{n N}=\frac{A_{0}^{2}\left(\sum_{i=1}^{n} k_{i}\right)^{2}}{n N} .
$$

Obviously, in the case when the signals in the channels are equal then the weighting coefficient are $k_{1}=k_{2}=\ldots=k_{i}=1$, the expression (4) is simplified:

$$
S N R=\frac{A_{0}^{2}\left(\sum_{i=1}^{n} k_{i}\right)^{2}}{n N}=\frac{A_{0}^{2}\left(\sum_{i=1}^{n} 1\right)^{2}}{n N}=\frac{A_{0}^{2} n^{2}}{n N}=\frac{A_{0}^{2} n}{N}=\frac{n S}{N} .
$$

From (5) it is clear that if $\frac{S}{N}$ - SNR one signal, by comparison SNR sum increases $n$ times.

In the case when there is a line of sight (los) path propagation, then the power of such a signal $P_{l o s}$ will be significantly higher than the power of the signals propagated as a result of the reflections $P_{r e f}[1,2,10]$. In this case, can represent the expression (3) in the form:

$$
S N R=\frac{\left(\sum_{i=1}^{n} A_{i}\right)^{2}}{n N}=\frac{\left(A_{\text {los }}+\sum_{i=1}^{n-1} A_{\text {ref } i}\right)^{2}}{n N}
$$

The signal level $A_{\text {ref } i}$ can be represented as $A_{\text {ref } i}=k_{i} A_{\text {los }}$, where $k_{i}$ is a weighting coefficient taking values from $0<k_{i} \leq 1$. (Obviously, in the case when $k_{i}=1$, for each term, we go to expression (5)). In view of the above, expression (6) can be written as: 


$$
\begin{gathered}
S N R=\frac{\left(A_{\text {los }}+\sum_{i=1}^{n-1} k_{i} A_{l o s}\right)^{2}}{n N}=\frac{A_{\text {los }}^{2}\left(1+\sum_{i=1}^{n-1} k_{i}\right)^{2}}{n N}=\frac{A_{\text {los }}^{2}\left(n\left(\frac{1}{n}+\frac{\sum_{i=1}^{n-1} k_{i}}{n}\right)\right)^{2}}{n N}= \\
=\frac{A_{\text {los }}^{2} n^{2}\left(\frac{1}{n}+\frac{\sum_{i=1}^{n-1} k_{i}}{n}\right)^{2}}{n N}=\frac{A_{l o s}^{2} n\left(\frac{1}{n}+\frac{\sum_{i=1}^{n-1} k_{i}}{n}\right)^{2}}{N}
\end{gathered}
$$

Denote $\sum_{i=1}^{n-1} k_{i}$ by $k_{\Sigma}$. Then $k_{\Sigma}$ varies $0<k_{\Sigma} \leq(n-1)$. The dependence of efficiency on $k_{\Sigma}$ is shown in figure 2 (for $n=100$ ).

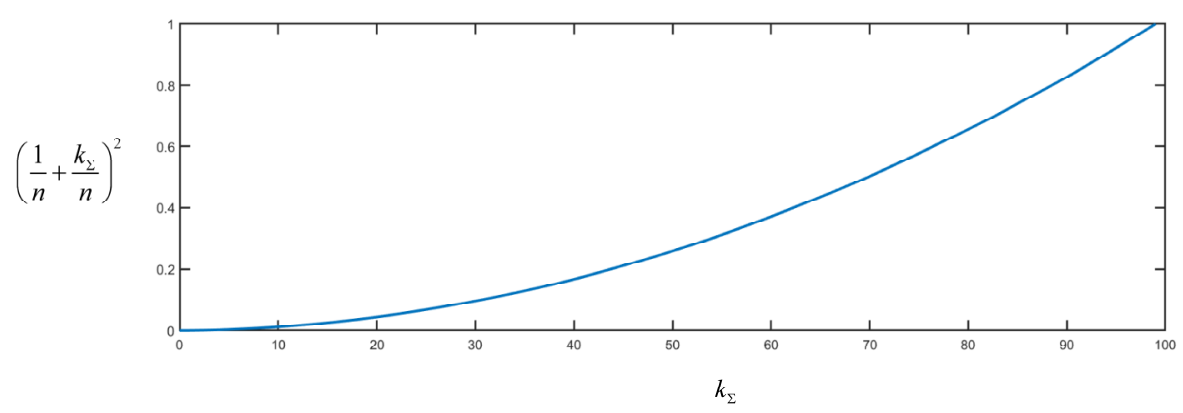

Fig. 2. Algorithm efficiency

Thus, on the basis of expression (7) and Figure 2, it can be obtained that in order to get the efficiency gain by SNR, it is necessary that $k_{\Sigma}>\left(1+\frac{n}{\sqrt{n}}\right)$. Given that this level has been defined to exclude signals filtration, it can be reduced using filters as shown in Figure 1.

\section{Conclusion}

In this article, the implementation of the received signal algorithm under conditions of multipath propagation of radio waves was considered. An expression of efficiency was obtained in terms of the SNR, with the result that the minimum signal level was detected to improve reception efficiency.

\section{References}

1. D. Chernyshov, V. Fedosov, MATEC Web of Conferences, 226, 05009 (2018)

2. D.Y. Chernyshov, V.I. Marchuk, S.V. Makov, A.Y. Minaev, Scientific and Technical Bulletin of the Volga region, 3, 140 (2019) 
3. J.G. Proakis, Digital signal processing: principles algorithms and applications (Prentice-Hall International, Inc., 1996)

4. T.S. Dhope, Faculty of Electrical Engineering and Computing, University of Zagreb, Croatia (2010)

5. T.B. Lavate, V.K. Kokate, A.M. Sapkal, 2010 Second International Conference on Computer and Network Technology, 308 (2010)

6. S. Chen, X. Li, Z. Shao, 2015 IEEE International Conference on Communication Problem-Solving (ICCP), 475 (2015)

7. R. Roy, T. Kailath, IEEE Transactions on Acoustics, Speech and Signal Processing, 32, 7, 984 (1989)

8. B.R. Levin, Theoretical Foundations of Statistical Radio Engineering (Soviet radio, 1969)

9. V.I. Tikhonov, Statistical Radio Engineering (Soviet radio, 1966)

10. Y. Karasawa, The Radio Science Bulletin, 302, 5 (2002) 